\title{
The influence of pregnancy on systemic immunity
}

\author{
Michael Pazos • Rhoda S. Sperling • Thomas M. Moran • \\ Thomas A. Kraus
}

Published online: 24 March 2012

(C) Springer Science+Business Media, LLC 2012

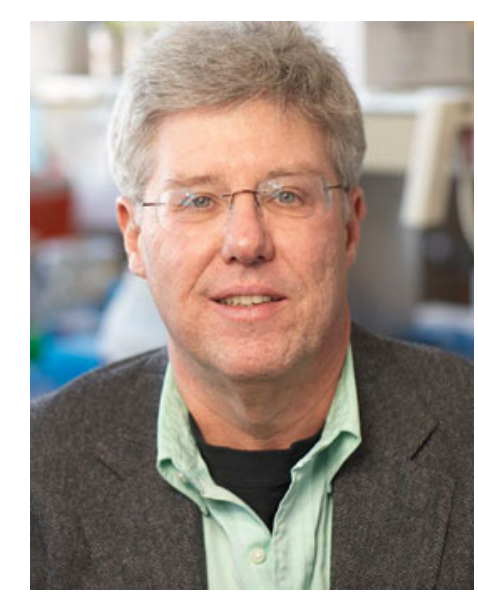

Thomas M. Moran

\begin{abstract}
Adaptations in maternal systemic immunity are presumed to be responsible for observed alterations in disease susceptibility and severity as pregnancy progresses. Epidemiological evidence as well as animal studies have shown that influenza infections are more severe during the second and third trimesters of pregnancy, resulting in greater morbidity and mortality, although the reason for this is still unclear. Our laboratory has taken advantage of 20 years of experience studying the murine immune response to respiratory viruses to address questions of altered immunity during pregnancy. With clinical studies and unique animal model systems, we are working to define the mechanisms responsible for altered immune responses to influenza infection during pregnancy and what roles hormones such as estrogen or progesterone play in these alterations.
\end{abstract}

Keywords Gestation · Immunology $\cdot$ Th2 $\cdot$ Fetus $\cdot$ Placenta $\cdot$ Pregnancy $\cdot$ Inflammation $\cdot$ Immunosuppression

\section{Reproductive hormones and immunity}

Although the term "reproductive immunology" simply refers to the interaction between the immune and reproductive systems, it is often used to describe the role of the immune system in reproduction. This is, admittedly, a very exciting area of research with many important applications. Infertility, pre-eclampsia, intrauterine growth restriction and pre-term labor all appear to have significant immune components [1,2].

From the immunologist's point of view, the interplay between steroid hormones and the immune system is intriguing. The systemic expression level of sex hormones differs according to age, gender and pregnancy status in

M. Pazos · T. M. Moran ( $\varangle)$. T. A. Kraus

Department of Microbiology, Mount Sinai School of Medicine,

1 Gustave L. Levy Place, Box 1124, New York, NY 10029, USA

e-mail: Thomas.Moran@mssm.edu

R. S. Sperling - T. A. Kraus

Department of Obstetrics, Gynecology, and Reproductive

Sciences, Mount Sinai School of Medicine, New York,

NY 10029, USA women. Most if not all immune cells express receptors for sex hormones. T cells, B cells, neutrophils, dendritic cells, monocytes and natural killer (NK) cells all have been shown to be transcriptionally regulated by estrogen [3-5]. The effect of steroid hormones on immune cells has been a target of extensive study [6]. Although a number of laboratories have reported on estrogen's effects at the molecular and cellular level, the role of hormones in a systemically coordinated immune response has been difficult to ascertain, let alone in the complex milieu of pregnancy. As gestation progresses, estradiol levels in the maternal serum can rise as much as 500 -fold against a backdrop of many maternal or placental-derived hormones that also fluctuate in serum. It is clear, though, that the influence of steroid hormones on immune cells occurs throughout the body and affects various organ systems, including the respiratory system during viral infection, and the nervous system during autoimmune attack. Alterations observed in some autoimmune disease states during pregnancy have been linked to estrogen signaling [7-9]. Relapse rates of multiple sclerosis decline during pregnancy and rheumatoid arthritis symptoms remit as pregnancy progresses, correlating strongly with estrogen levels 
$[10,11]$. Due to the striking remission in autoimmune symptoms, pregnancy is often described as a time of pure immunosuppression.

\section{Influenza infection during pregnancy}

More evidence for the immunosuppressive hypothesis of pregnancy is found in the increased disease severity to some infectious pathogens. Increase risk of severe Varicella zoster [12], SARS [13, 14], Listeria monocytogenes [15], Plasmodium falciparum [16] infections have all been observed during pregnancy. Since the 1918 influenza pandemic, it has been apparent that pregnant women suffer more severe complications from influenza infections than non-pregnant women (Fig. 1). In 1918, the death rate for men and women of child-bearing age infected with influenza climbed to about $1 \%$ [17]. For pregnant women, the rate was $27 \%$, and in cases complicated with pneumonia, over $50 \%$ [18]. This pattern is borne out by every major pandemic of the last century, including the recent 2009 H1N1 pandemic [19-21]. Furthermore, women in the second and third trimesters have higher mortality and morbidity rates than first trimester women. Similar observations have been made in response to seasonal strains, albeit to a lesser degree [22].

As is the case for many illnesses that affect pregnant women, the burden of an influenza infection is twofold, with both fetal and maternal complications. Maternal infections have been associated with spontaneous loss and pre-term delivery [23, 24]. Epidemiological evidence as well as animal model studies suggest that maternal influenza infection may increase the risk schizophrenia and autism in offspring [25-28]. These studies suggest that, although the virus is restricted to the maternal lungs, inflammatory cytokines such as IL-6 may compromise the

Increased mortality or morbidity compared to nonpregnant

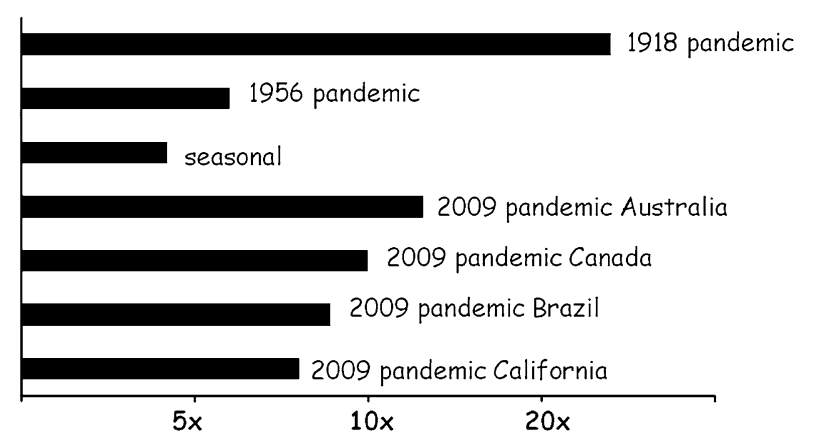

Fig. 1 Increased morbidity and mortality to influenza in pregnant women compared to the general population. Statistics were taken from refs. [18-23] fetus. This is no small problem. There are six million pregnant women per year in the United States and it is estimated that $\sim 10 \%$ will be infected by influenza [29]. In pandemic years, the number will increase. Protecting both the mother and the fetus has enormous public health importance.

\section{Theories of immune response during pregnancy}

Because of the autoimmune alterations and changes in infectious disease severity that occur during pregnancy, two theories of immune alteration have become prevalent. The first is that there is a general immune suppression that reduces the likelihood of an antigen-specific response against the semi-allogenic fetus. The origin of the theory from the great transplant immunologist Peter Medawar, actually predates the epidemiological data and much of what we now know about immunology and placental design. Although exact mechanisms of fetal tolerance are still being uncovered, it is clear that the uterus/placenta represents an immune privileged site and that there are multiple mechanisms for keeping local inflammation and specific T-cell response under control [30].

The second pervasive theory is that there is an extensive Th2 bias that occurs and is vital to the success of the pregnancy. This was first published 20 years ago and is largely based upon evidence gathered from mouse experimental data and, importantly, at the maternal/fetal interface. Yet researchers have speculated that a shift to Th2 immunity is responsible for the altered responses in the periphery to respiratory viral infections [31] or autoantigens [32, 33]. Most evidence supporting a Th2 shifts derives from studies of material/fetal interface rather than systemic immunity. Although inflammatory events have been shown to be important at critical times at the beginning and end of gestation, for the most part, the uterine environment is biased toward Th2 [34]. Arguments for a Th2 bias in the periphery are much more contentious [35].

\section{Is there a systemic immunosuppression during pregnancy?}

Alterations occur in the systemic immune system during pregnancy that could explain both the reduction in autoimmune attacks and the increased severity to influenza infections. We have found a decrease in the total number of $\mathrm{CD} 3+\mathrm{T}$ cells in blood. The surge in estrogen and progesterone at the end of the first trimester results in a reversible thymic involution during pregnancy, which is likely responsible for this decrease in both $\mathrm{CD} 4$ and $\mathrm{CD} 8 \mathrm{~T}$ cells [36, 37]. However, the T-cell defect is more than just 
a reduction in newly released thymocytes. TSST-stimulated CD45RA+ (naïve) $\mathrm{T}$ cells isolated from the periphery during pregnancy show a profound decrease in both Th1 and Th 2 cytokines when compared to cells from the same subjects collected in the post-partum period [38]. Therefore, in addition to a reduction in $\mathrm{T}$ cell numbers in the blood during pregnancy, the cells have broadly reduced activity when stimulated without any apparent bias toward a Th2 phenotype.

NK cells are critically important in early pregnancy. Uterine NK ( $\mathrm{uNK}$ ) cells mediate trophoblast invasion and placental redesign early in gestation [39]. Up to $70 \%$ of the decidual stromal leukocytes are NK cells, and depletion studies have shown them to be vital to the pregnancy. The current understanding is that after the placenta is established, the role of NK cells is less important, and their numbers in the decidua decrease [40]. Interestingly, in our study, NK cells numbers in the periphery begin decrease as well after 20-week gestation [38].

Peripheral NK cells make up about $10-15 \%$ of the circulating leukocytes; about $85 \%$ are $\mathrm{CD} 56^{\mathrm{dim}} \mathrm{CD} 16+$. Their primary function in immunity is to respond to infection by secreting cytokines such as IFN $\gamma$ and potentiating the adaptive response. These cells are also cytotoxic, and if stimulated with an activating ligand, will lyse target cells. After 20 weeks of gestation, these cells decrease in number in the maternal blood and similar to what was observed in naïve $\mathrm{T}$ cells, also have reduced activity. Their secretion of cytokines, either in the unstimulated state or when stimulated with either IL-2 (unpublished data) or a combination of IL-12 and IL-15, is significantly impaired, impacting their ability to amplify adaptive immune responses. All NK cell subsets have activating and inhibitory receptors that bind to soluble ligands, and although peripheral NK cells perform different functions than uNK cells, their activity during pregnancy might reflect the uterine environment.

B cells are also affected by pregnancy. Medina and Kincade have shown convincingly that estrogen reduces B-cell lymphopoiesis during pregnancy [41]. Grimaldi et al. [42] have reported on the effects of estrogen on B-cell activity. We reported that this reduction is reflected by week 34 when peripheral CD19+ cells are significantly reduced in the blood. In mouse studies, it has been reported that in response to influenza infection antibody titers are lower in pregnant mice [43]. We have confirmed this in our laboratory (Pazos unpublished data).

These observations may provide a more mechanistic understanding of autoimmune remission and of increased pathogenesis of influenza infections during pregnancy. The decrease in T-cell activity is consistent with the reduced flares in RA and MS. Even though we have not found a $\mathrm{Th} 2$ bias in cytokine secretion in serum or in T-cell supernatants, a profound reduction in the release of highly inflammatory Th1 cytokines occurs during the later stages of pregnancy. This could conceivably shift the antigenspecific autoimmune response away from a pathogenic Th1 response. Although the role of NK cells in the immunopathology of RA and MS is still unclear, the alteration in function of NK cells during pregnancy could be an intriguing component of the altered phenotype seen in these diseases during pregnancy $[44,45]$.

In influenza infection, the epidemiological data suggest increased severity of infection, but an increase in susceptibility to infection has not been documented. The data suggest that once an infection is established, the risk of increased severity of disease is high, especially in the second and third trimesters.

This fits well with the decrease in adaptive immunity. CD4 and CD8 cells are important for influenza viral clearance, and the loss of function, along with the decreased $\mathrm{T}$ cells in the periphery perhaps, could delay recovery. NK cells have been shown to be important for influenza virus response, mostly as cytokine secretors and mediators of adaptive responses and not as cytotoxic cells [46]. Therefore, the immune responses that are suppressed are those involved in recovery as opposed to the prevention of infection.

\section{How do women survive pregnancy?}

The evolutionary adaptation to vivipary would not have been successful if it allowed the mother to be broadly vulnerable to infectious disease. Along with the decrease in T-cell and NK cell function and numbers, there is a concomitant increase in some aspects of innate immunity. An increase in phagocytic immune cells, including monocytes, dendritic cells and neutrophils, is observed in maternal blood from as early as gestational week 20-week 36. [38] Increased phagocytic activity, along with increases in number of circulating PMNs, has been reported, (reviewed in [47]). $\alpha$-Defensins, which are polypeptides that can inhibit microbes in a variety of ways [48], are increased in the serum throughout pregnancy [38].

Pregnancy might further be a time of high IFN sensitivity. We have found plasmacytoid DCs to be increased in the periphery throughout our study. We noted increased transcription of interferon-stimulated genes (ISGs) from both TSST-stimulated $\mathrm{T}$ cells and monocytes during pregnancy. While we have not found a significant increase in type 1 IFN in the serum during pregnancy, we have noted slight elevations up to 20-week gestation (unpublished observations). One does not typically associate pregnancy as a time of increased protection from viral infection, but the bolstered immune surveillance associated 
with an increase in phagocytic cells, antimicrobial peptides and enhanced ISG expression might diminish the probability of infection. This does not conflict with the epidemiological data that merely suggest that after certain viral infections are established, recovery might be hindered.

Taken together, these alterations are summarized in Fig. 2. Immunity during pregnancy is altered but on the whole does not leave the mother without defense against pathogenic microbes. It is possible that pregnant women survive by an increased emphasis on the prevention of infection. Increases in monocytes, DCs, neutrophils, serum defensins may offset the downregulation of T cells and NK cells and B cells. We have hypothesized that maternal immunity strives to decrease inflammatory events so as not to expose the fetus to potentially dangerous inflammatory signals. However, in exchange for the protection of the fetus, the mother sacrifices efficient viral clearance, which could prove tragic if the exposure level is too great to be contained by the bolstered innate immune system. We have called this a shift to defensive immunity to represent a bias of the mother to defend herself and the fetus without the full activity of responding $\mathrm{T}$ and NK cells. Inflammation is decreased to protect the fetus from any untoward effects of inflammatory cytokines in the blood.

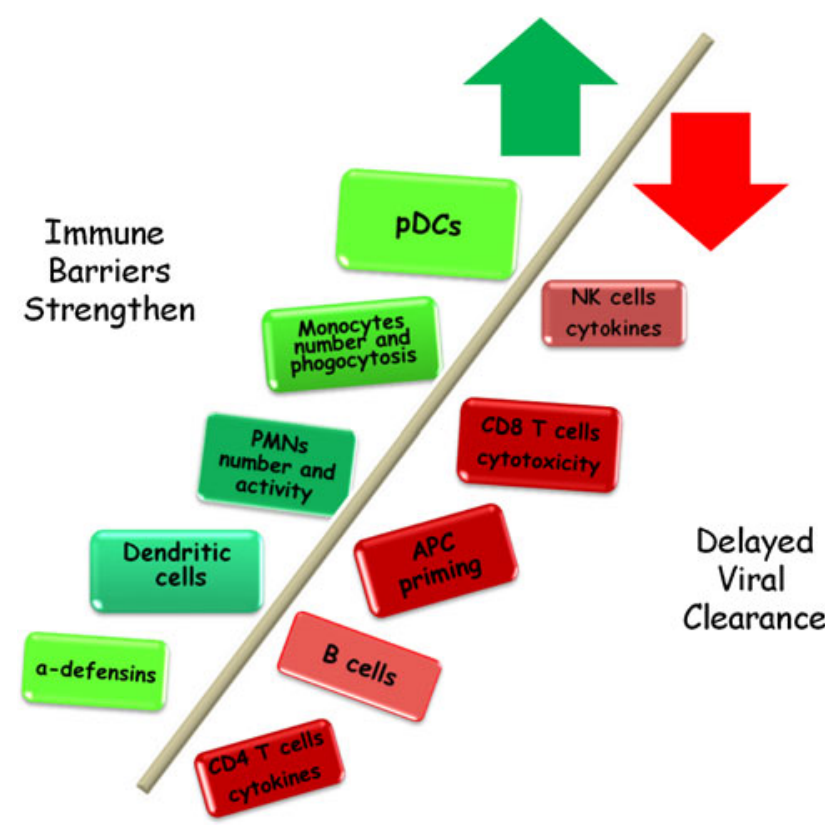

Fig. 2 Model of immune alterations during pregnancy. Strengthening of immune barrier functions includes increased phagocytosis, PMN activity, serum defensins and pDCs, while adaptive immunity, including NK cell cytokine secretion, T-cell activity and possibly B-cell activity, is weakened

\section{Serum cytokine alterations during pregnancy}

During an influenza infection, cytokines are released into the blood starting on day 2 post-infection. This has been reported in human experimental infections as well as in mouse models. We have reported that these serum cytokines play a large role in the immune responses [49]. But these cytokines and chemokines are also present in the serum when there is no active infection. There have been reports of these cytokines being present at very stable concentrations for over 2 years [50].

We performed a longitudinal pregnancy study and tracked modulation of serum cytokines. By analyzing post-partum samples as non-pregnant controls, we noted remarkable variation in constitutively expressed cytokine levels between individuals [51]. Despite the variation between women, most cytokines were remarkably stable between samples collected at 6 weeks and 6 months post-partum with an ICC value of over 0.6 , many over 0.8 . Others have noted similar stability $[50,52,53]$. The observation that individuals have different but stable cytokine fingerprints is intriguing. Not only can these patterns be used as possible biomarkers for disease susceptibility but they might be able to predict the protein content of immune cells. For example, NK cells have a welldescribed IL-12/IFN $\gamma$ loop where IL-12 binding by NK cells leads to enhanced secretion of IFN $\gamma$ from stimulated cells. In our cohort, NK cells from subjects with the highest serum concentrations of IL-12 showed a statistically significant increase in IFN $\gamma$, demonstrating that the IL-12 in serum was active and influenced the activation status of the circulating NK cells.

When we sampled serum throughout pregnancy, we found that several cytokines had dramatically altered expression levels. For most subjects, VEGF-A levels decreased significantly, as did IFN $\gamma$, MCP-1 and Eotaxin. VEGF is associated with many inflammatory diseases such as RA and MS, and it is interesting to note its decrease during pregnancy correlates with the reduction in symptoms found in pregnant MS patients. IFN $\gamma$ is also reduced in serum during pregnancy, as is MCP-1 and Eotaxin. $\mathrm{TNF} \alpha$ and G-CSF rise during pregnancy (Fig. 3). Generally, the serum alterations reflect our overall view of the pregnant immune phenotype. IFN $\gamma$ and VEGF, inflammatory cytokines, are reduced, while cytokines that induce phagocytic cell recruitment or activity (TNF $\alpha, \mathrm{G}-\mathrm{CSF})$ increase. Alterations were consistent but did not follow a Th1 or Th2 phenotype.

\section{Vaccination responses during pregnancy}

In order to protect pregnant women and their newborns, routine vaccination of all pregnant women throughout 


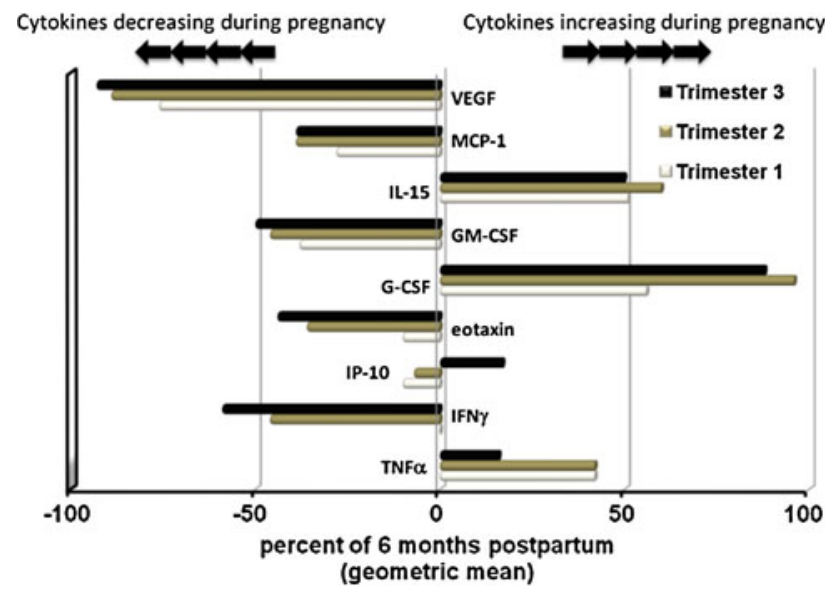

Fig. 3 Cytokine alterations during pregnancy. Cytokines were measured by multiplex ELISA three times during pregnancy and compared to 6th month post-partum. Data were first published in ref. [50]

gestation with trivalent inactivated influenza vaccine (TIV) has been recommended. Vaccination rates are improving but suboptimal [54]. Although thorough clinical studies have been few, maternal vaccination has been thought to be a safe and effective tool in the effort to protect the mother and child from severe influenza infections [55, 56].

In light of the alterations in systemic immunity during pregnancy, we evaluated immunologic responses to inactivated influenza vaccine in a cohort of antepartum and postpartum women receiving an influenza vaccination as part of their clinical care. The objective was to estimate the effects of gestational age and other maternal factors on immunologic responses to influenza vaccination. We recruited over 230 women over the 4-year study and measured the seroconversion rate of each influenza A strain in the seasonal trivalent inactivated influenza (TIV) vaccine. In 2009, we adapted our protocol to include the measurement of the pandemic $\mathrm{H} 1 \mathrm{~N} 1$ response, which was administered in a separate vaccine. We identified no statistically significant differences in

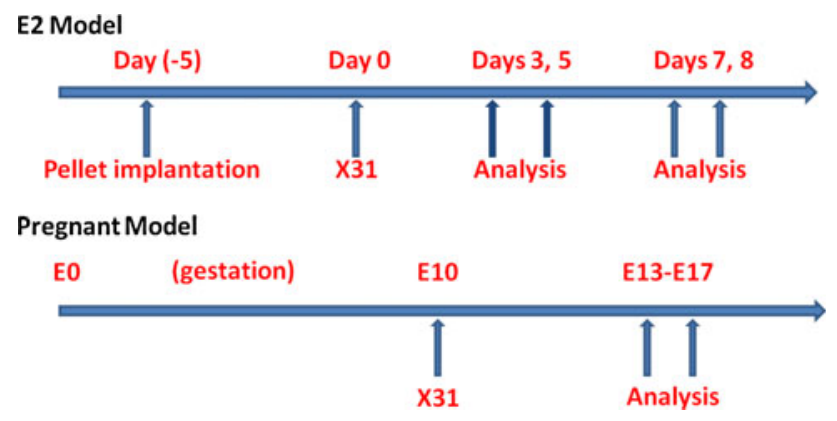

Fig. 4 Experimental schema. Non-pregnant mice are implanted with hormone or placebo pellet. Five days later, mice are infected with influenza X31 or mock infected. (Top) Pregnant mice are infected with X31 on E10 (Bottom). Starting at three days post-infection, mice are analyzed for cytokine and chemokine production and cell migration seroconversion rates according to trimester of vaccination. However, we observed somewhat lower rates of seroconversion for women vaccinated in the first trimester and for obese women. In a multivariable model, higher baseline antibody levels and prior year flu vaccination were both significantly associated with reduced odds of seroconversion (Sperling et al. in press). The downregulation of immunity that occurs through thymic involution and B-cell lymphopoiesis apparently does not hinder the antibody response to influenza vaccine. Whether this would be true for other vaccines that might rely more on primary responses is not known and should be explored (Fig. 4).

\section{Murine models of influenza infection during pregnancy}

Epidemiological studies of pregnant women exposed to influenza have proven to be a useful source of information, but in order to dissect the biological mechanisms at play, the use of manipulable animal models is necessary. Murine models of influenza virus infection are well established. Our laboratory uses an aerosol infection chamber model to study innate and adaptive immune responses to influenza virus in mice. Aerosol inoculations result in highly reproducible infections, which replicate clinical descriptions of human influenza infection more closely than intranasal inoculations [57, 58].

Using this system, we have made observations that have shaped our understanding of DC responses to respiratory viruses [57, 59] and the effects of antiviral immunity on distal organs [49]. Recently, we extended these observations to show how type I interferon sensitivity differentially impacts DC subsets that migrate from the lung to the lymph nodes after infection [60].

In order to adapt this model to the study of influenza during pregnancy, mice must demonstrate key clinical correlates similar to what has been observed in studies of human reproductive biology. Although biological differences exist in placentation between mice and human [61], some early work in mouse models demonstrates that there is reason to believe that mechanisms impacting immunity in pregnancy are conserved. It has been demonstrated that infection during pregnancy impacts litter size and health [62]. Pregnant mice experience increased mortality that correlates with late-term pregnancy [63], analogous to observations in the final trimester of human pregnancy. These observations have recently been confirmed using the 2009 pandemic H1N1 Influenza A strain [43]. Pregnant mice have altered or delayed cytokine production similar to observations in pregnant women [43]. Even though these models have been used for at least 50 years, the mechanism of increased morbidity and mortality in pregnant mice remains unclear. 
To help answer this question, we adapted our aerosolbased model to study influenza virus infection during pregnancy. Because gestation in mice is short, very strict timing must be used to reproduce infection during late-term pregnancy. Animals are infected at E10 and analyzed throughout infection to the end of term.

Animal models also afford additional flexibility to thoroughly investigate the various contributors to altered immune responses during pregnancy. Hormone and hormone inhibitor treatments in animal models have successfully been used to describe the role estrogens play in modulating Multiple Sclerosis [9, 64], a phenomenon originally observed in pregnant women with the disease. We established a second parallel model in non-pregnant mice implanted with hormone pellets. The biodegradable pellets are implanted under the skin and release a steady stream of sex hormone for 21 days. While pregnancy is a complicated process with regularly fluctuating hormone levels, using this model, we are able to control a single variable and contrast these observations with analogous results in the pregnancy model. Using these two models, we found that high expression of estrogen appears to play a significant role during influenza infection. It appears to have protective effects by suppressing inflammatory responses [65], and weight loss is reduced in the estrogenpelleted mice compared to placebo. However, estrogen also reduces CD8 T-cell cytotoxic activity both directly and as a result of less efficient APC activation. Since CD8 T cells function to kill virus-infected cells, this is likely to be responsible for the delayed viral clearance found during pregnancy. NK cell responses are also reduced during influenza infection, which likely contributes to the delay of adaptive immunity (Pazos et al. manuscript under review).

The potential for pregnancy models in mice is very exciting when considering the tools available for continued study. Transgenic mice knocked out for components of hormone signaling pathways or immune responses are available. ER $\beta \mathrm{KO}$ mice have been studied in MS models [66], yielding detailed mechanisms that provide opportunity for targeted therapeutics. Depletion studies have demonstrated the critical role for regulatory $\mathrm{T}$ cells in pregnancy [67]. Additionally, syngeneic and allogeneic matings can be used to investigate the regulation of antigen-specific responses during pregnancy $[68,69]$. There is no shortage of tools in the mouse to be leveraged that can identify critical mechanisms of immune modulation during pregnancy.

\section{Future directions}

As we learn more about the immune alterations occurring during pregnancy, it becomes clear that pregnancy represents more than a systemic immune suppression. There does exist strong evidence that adaptive immune responses are weakened during pregnancy, potentially explaining reduced viral clearance, and alleviated symptoms in some autoimmune diseases. While a strong innate immune response seems at odds with late-term pregnancy, our data suggest that boosted defensive immune mechanisms such as increased numbers of phagocytes and elevated defensin levels may represent a compensatory immune mechanism to protect the pregnant mother and child. This may imply reduced susceptibility to initial infection and represents an interesting avenue for future research.

Mechanisms of estrogen and progesterone modulation on individual immune components have been extensively studied in vitro, but it is still unclear how these pieces integrate in a live host. A detailed understanding of the mechanisms and contributions of individual steroid hormones to the modulation observed during pregnancy would be of overwhelming clinical value. Animals models provide the controlled experimental conditions and detailed sample collection that is impossible in epidemiological studies.

A full understanding of the contribution of steroid hormones to immunity may yield novel targeted therapeutic options for pregnant women and their children that may be suffering from significant pregnancy-associated morbidities. It may also yield novel therapeutic avenues of immune modulation for the treatment for various diseases in nonpregnant individuals. At the intersection of reproductive endocrinology and immunology, there exist a series of testable hypotheses regarding the manipulation of human immunity that may provide significant clinical value.

\section{References}

1. Brosens I, et al. The "Great Obstetrical Syndromes" are associated with disorders of deep placentation. Am J Obstet Gynecol. 2011;204(3):193-201.

2. Makrigiannakis A, et al. Recent advances in understanding immunology of reproductive failure. J Reprod Immunol. 2011;90(1): 96-104.

3. Cvoro A, et al. Selective estrogen receptor-beta agonists repress transcription of proinflammatory genes. J Immunol. 2008;180(1): 630-6.

4. Lambert KC, et al. Estrogen receptor alpha (ERalpha) deficiency in macrophages results in increased stimulation of $\mathrm{CD} 4+\mathrm{T}$ cells while 17beta-estradiol acts through ERalpha to increase IL-4 and GATA-3 expression in CD4+ T cells independent of antigen presentation. J Immunol. 2005;175(9):5716-23.

5. Pierdominici $M$, et al. Estrogen receptor profiles in human peripheral blood lymphocytes. Immunol Lett. 2010;132(1-2): 79-85.

6. Straub RH. The complex role of estrogens in inflammation. Endocr Rev. 2007;28(5):521-74. 
7. Fairweather D, Frisancho-Kiss S, Rose NR. Sex differences in autoimmune disease from a pathological perspective. Am J Pathol. 2008;173(3):600-9.

8. Jansson L, Olsson T, Holmdahl R. Estrogen induces a potent suppression of experimental autoimmune encephalomyelitis and collagen-induced arthritis in mice. J Neuroimmunol. 1994;53(2):203-7.

9. Elloso MM, et al. Suppression of experimental autoimmune encephalomyelitis using estrogen receptor-selective ligands. J Endocrinol. 2005;185(2):243-52.

10. Confavreux $\mathrm{C}$, et al. Rate of pregnancy-related relapse in multiple sclerosis. Pregnancy in Multiple Sclerosis Group. N Engl J Med. 1998;339(5):285-91.

11. Klipple GL, Cecere FA. Rheumatoid arthritis and pregnancy. Rheum Dis Clin North Am. 1989;15(2):213-39.

12. Harger $\mathrm{JH}$, et al. Risk factors and outcome of varicella-zoster virus pneumonia in pregnant women. J Infect Dis. 2002;185(4): 422-7.

13. Stockman LJ, et al. SARS during pregnancy, United States. Emerg Infect Dis. 2004;10(9):1689-90.

14. Wong SF, Chow KM, de Swiet M. Severe acute respiratory syndrome and pregnancy. BJOG. 2003;110(7):641-2.

15. Braden CR. Listeriosis. Pediatr Infect Dis J. 2003;22(8):745-6.

16. Shulman CE, Dorman EK. Importance and prevention of malaria in pregnancy. Trans R Soc Trop Med Hyg. 2003;97(1):30-5.

17. Taubenberger JK, Morens DM. 1918 Influenza: the mother of all pandemics. Emerg Infect Dis. 2006;12(1):15-22.

18. Harris J. Influenza occurring in pregnant women. J Am Med Assoc. 1919;72(14):978-80.

19. Greenberg M, et al. Maternal mortality in the epidemic of Asian influenza, New York City, 1957. Am J Obstet Gynecol. 1958; 76(4):897-902.

20. Freeman DW, Barno A. Deaths from Asian influenza associated with pregnancy. Am J Obstet Gynecol. 1959;78:1172-5.

21. Klein SL, et al. The impact of sex, gender and pregnancy on 2009 H1N1 disease. Biol Sex Differ. 2010;1(1):5.

22. Neuzil KM, et al. Impact of influenza on acute cardiopulmonary hospitalizations in pregnant women. Am J Epidemiol. 1998; 148(11):1094-102.

23. Rasmussen SA, Jamieson DJ, Bresee JS. Pandemic influenza and pregnant women. Emerg Infect Dis. 2008;14(1):95-100.

24. Omer SB, et al. Maternal influenza immunization and reduced likelihood of prematurity and small for gestational age births: a retrospective cohort study. PLoS Med. 2011;8(5):e1000441.

25. Patterson PH. Neuroscience. Maternal effects on schizophrenia risk. Science. 2007;318(5850):576-7.

26. Brown AS, Susser ES. In utero infection and adult schizophrenia. Ment Retard Dev Disabil Res Rev. 2002;8(1):51-7.

27. Smith SE, et al. Maternal immune activation alters fetal brain development through interleukin-6. J Neurosci. 2007;27(40): 10695-702.

28. Fatemi SH, et al. Prenatal viral infection in mouse causes differential expression of genes in brains of mouse progeny: a potential animal model for schizophrenia and autism. Synapse. 2005;57(2):91-9.

29. Mak TK, et al. Influenza vaccination in pregnancy: current evidence and selected national policies. Lancet Infect Dis. 2008;8(1):44-52.

30. Erlebacher A. Why isn't the fetus rejected? Curr Opin Immunol. 2001;13(5):590-3.

31. Jamieson DJ, Theiler RN, Rasmussen SA. Emerging infections and pregnancy. Emerg Infect Dis. 2006;12(11):1638-43.

32. Wilder RL. Hormones, pregnancy, and autoimmune diseases. Ann NY Acad Sci. 1998;840:45-50.

33. Ostensen M, Villiger PM. Immunology of pregnancy-pregnancy as a remission inducing agent in rheumatoid arthritis. Transpl Immunol. 2002;9(2-4):155-60.
34. Mor G, Cardenas I. The immune system in pregnancy: a unique complexity. Am J Reprod Immunol. 2010;63(6):425-33.

35. Chaouat G. The Th1/Th2 paradigm: still important in pregnancy? Semin Immunopathol. 2007;29(2):95-113.

36. Zoller AL, Schnell FJ, Kersh GJ. Murine pregnancy leads to reduced proliferation of maternal thymocytes and decreased thymic emigration. Immunology. 2007;121(2):207-15.

37. Clarke AG, Kendall MD. The thymus in pregnancy: the interplay of neural, endocrine and immune influences. Immunol Today. 1994;15(11):545-51.

38. Kraus TA, et al. Characterizing the pregnancy immune phenotype: results of the Viral Immunity and Pregnancy (VIP) Study. J Clin Immunol. 2011;32:300-11.

39. Fatemi SH, et al. Maternal infection leads to abnormal gene regulation and brain atrophy in mouse offspring: implications for genesis of neurodevelopmental disorders. Schizophr Res. 2008;99(1-3):56-70.

40. Williams PJ, et al. Decidual leucocyte populations in early to late gestation normal human pregnancy. J Reprod Immunol. 2009; 82(1):24-31.

41. Medina KL, Smithson G, Kincade PW. Suppression of B lymphopoiesis during normal pregnancy. J Exp Med. 1993;178(5):1507-15.

42. Grimaldi CM, et al. Estrogen alters thresholds for B cell apoptosis and activation. J Clin Invest. 2002;109(12):1625-33.

43. Chan KH, et al. Wild type and mutant 2009 pandemic influenza A (H1N1) viruses cause more severe disease and higher mortality in pregnant BALB/c mice. PLoS ONE. 2010;5(10):e13757.

44. Morandi B, et al. Role of natural killer cells in the pathogenesis and progression of multiple sclerosis. Pharmacol Res. 2008; 57(1):1-5.

45. Conigliaro P, et al. Emerging role for NK cells in the pathogenesis of inflammatory arthropathies. Autoimmun Rev. 2011;10(10):577-81.

46. Jost $\mathrm{S}$, et al. Changes in cytokine levels and NK cell activation associated with influenza. PLoS ONE. 2011;6(9):e25060.

47. Priddy KD. Immunologic adaptations during pregnancy. J Obstet Gynecol Neonatal Nurs. 1997;26(4):388-94.

48. Klotman ME, Chang TL. Defensins in innate antiviral immunity. Nat Rev Immunol. 2006;6(6):447-56.

49. Hermesh T, et al. Antiviral instruction of bone marrow leukocytes during respiratory viral infections. Cell Host Microbe. 2010; 7(5):343-53.

50. Ho GY, et al. Variability of serum levels of tumor necrosis factoralpha, interleukin 6 , and soluble interleukin 6 receptor over 2 years in young women. Cytokine. 2005;30(1):1-6.

51. Kraus TA, et al. Peripheral blood cytokine profiling during pregnancy and post-partum periods. Am J Reprod Immunol. 2010;64(6):411-26.

52. Wong HL, et al. Reproducibility and correlations of multiplex cytokine levels in asymptomatic persons. Cancer Epidemiol Biomarkers Prev. 2008;17(12):3450-6.

53. Knudsen LS, et al. Pre-analytical and biological variability in circulating interleukin 6 in healthy subjects and patients with rheumatoid arthritis. Biomarkers. 2008;13(1):59-78.

54. Ding $\mathrm{H}$, et al. Influenza vaccination coverage among pregnant women-National 2009 H1N1 Flu Survey (NHFS). Am J Obstet Gynecol. 2011;204(6 Suppl 1):S96-106.

55. Poehling KA, et al. Impact of maternal immunization on influenza hospitalizations in infants. Am J Obstet Gynecol. 2011;204(6 Suppl 1):S141-8.

56. Blanchard-Rohner G, Siegrist CA. Vaccination during pregnancy to protect infants against influenza: why and why not? Vaccine. 2011;29(43):7542-50.

57. Moltedo B, et al. Cutting edge: stealth influenza virus replication precedes the initiation of adaptive immunity. J Immunol. 2009;183(6):3569-73. 
58. Treanor $\mathrm{J}$, et al. Intranasal administration of a proteosomeinfluenza vaccine is well-tolerated and induces serum and nasal secretion influenza antibodies in healthy human subjects. Vaccine. 2006;24(3):254-62.

59. Lopez CB, et al. TLR-independent induction of dendritic cell maturation and adaptive immunity by negative-strand RNA viruses. J Immunol. 2004;173(11):6882-9.

60. Moltedo B, et al. Unique type I interferon responses determine the functional fate of migratory lung dendritic cells during influenza virus infection. PLoS Pathog. 2011;7(11):e1002345.

61. Moffett A, Loke C. Immunology of placentation in eutherian mammals. Nat Rev Immunol. 2006;6(8):584-94.

62. Siem RA, et al. Influenza virus infections in pregnant mice. J Neuropathol Exp Neurol. 1960;19:125-9.

63. Williams K, Mackenzie JS. Influenza infections during pregnancy in the mouse. J Hyg (Lond). 1977;79(2):249-57.

64. Kim S, et al. Estriol ameliorates autoimmune demyelinating disease: implications for multiple sclerosis. Neurology. 1999; 52(6):1230-8.
65. Robinson DP, et al. Elevated 17beta-estradiol protects females from influenza A virus pathogenesis by suppressing inflammatory responses. PLoS Pathog. 2011;7(7):e1002149.

66. Tiwari-Woodruff S, Voskuhl RR. Neuroprotective and antiinflammatory effects of estrogen receptor ligand treatment in mice. J Neurol Sci. 2009;286(1-2):81-5.

67. Aluvihare VR, Kallikourdis M, Betz AG. Regulatory T cells mediate maternal tolerance to the fetus. Nat Immunol. 2004;5(3): 266-71.

68. Tafuri A, et al. T cell awareness of paternal alloantigens during pregnancy. Science. 1995;270(5236):630-3.

69. Kahn DA, Baltimore D. Pregnancy induces a fetal antigen-specific maternal $\mathrm{T}$ regulatory cell response that contributes to tolerance. Proc Natl Acad Sci USA. 2010;107(20):9299-304. 